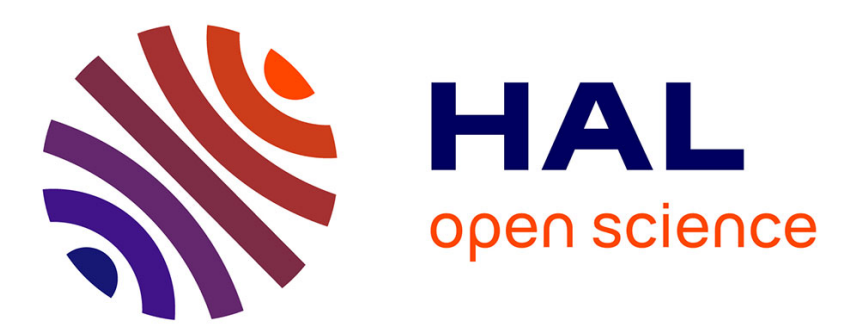

\title{
Acoustic streaming produced by a cylindrical bubble undergoing volume and translational oscillations in a microfluidic channel
}

Alexander A. Doinikov, Thomas Combriat, Pierre A Thibault, Philippe

Marmottant

\section{To cite this version:}

Alexander A. Doinikov, Thomas Combriat, Pierre A Thibault, Philippe Marmottant. Acoustic streaming produced by a cylindrical bubble undergoing volume and translational oscillations in a microfluidic channel. Physical Review E , 2016, 94 (3), 10.1103/PhysRevE.94.033109 . hal-02176425

\section{HAL Id: hal-02176425 \\ https://hal.science/hal-02176425}

Submitted on 8 Jul 2019

HAL is a multi-disciplinary open access archive for the deposit and dissemination of scientific research documents, whether they are published or not. The documents may come from teaching and research institutions in France or abroad, or from public or private research centers.
L'archive ouverte pluridisciplinaire HAL, est destinée au dépôt et à la diffusion de documents scientifiques de niveau recherche, publiés ou non, émanant des établissements d'enseignement et de recherche français ou étrangers, des laboratoires publics ou privés. 


\title{
Acoustic streaming produced by a cylindrical bubble undergoing volume and translational oscillations in a microfluidic channel
}

\author{
Alexander A. Doinikov, Thomas Combriat, Pierre Thibault, and Philippe Marmottant \\ LIPhy, UMR 5588, CNRS/Université Grenoble-Alpes, Grenoble F-38401, France
}

(Received 23 June 2016; published 14 September 2016)

\begin{abstract}
A theoretical model is developed for acoustic streaming generated by a cylindrical bubble confined in a fluid channel between two planar elastic walls. The bubble is assumed to undergo volume and translational oscillations. The volume oscillation is caused by an imposed acoustic pressure field and generates the bulk scattered wave in the fluid gap and Lamb-type surface waves propagating along the fluid-wall interfaces. The translational oscillation is induced by the velocity field of an external sound source such as another bubble or an oscillatory fluid flow. The acoustic streaming is assumed to result from the interaction of the volume and the translational modes of the bubble oscillations. The general solutions for the linear equations of fluid motion and the equations of acoustic streaming are calculated with no restrictions on the ratio between the viscous penetration depth and the bubble size. Approximate solutions for the limit of low viscosity are provided as well. Simulations of streamline patterns show that the geometry of the streaming resembles flows generated by a source dipole, while the vortex orientation is governed by the driving frequency, bubble size, and the distance of the bubble from the source of translational excitation. Experimental verification of the developed theory is performed using data for streaming generated by bubble pairs.
\end{abstract}

DOI: 10.1103/PhysRevE.94.033109

\section{INTRODUCTION}

In recent years, the dynamics of acoustically excited cylindrical bubbles has attracted considerable attention in the context of research on microfluidic systems [1-3]. Steady recirculating flows produced by such bubbles, referred to as acoustic streaming [4-6], are essential to a number of technical developments, such as particle trapping [7-9], size-selective sorting [10,11], shear force actuation [12], and micromixing [13-16]. Consequently, a large amount of studies have been devoted to the streaming generated by acoustically excited spherical bubbles [17-28]. Theoretical developments on this subject show that the modeling of acoustic streaming is a challenging mathematical problem, which requires a great effort to bring theoretical calculations close to situations of practical interest. The literature on the streaming generated by cylindrical bubbles is much less extensive. Raney et al. [29] developed a theoretical treatment of the streaming around a solid cylinder immersed in an oscillating fluid. It gives an insight into the structure of steady flows caused by the translational type of motion of a cylindrical object. Rallabandi and co-workers [30,31] developed a theory for streaming flows induced by a harmonically oscillating semicylindrical bubble attached to a rigid wall. In this case, the acoustic streaming is caused by the mixed-mode interaction of the volume (breathing) mode and shape modes. In a different context, Mekki-Berrada et al. [32] have recently shown that the acoustic streaming generated by cylindrical bubbles in a microfluidic channel also results from the mixed-mode interaction but this interaction occurs between the volume and translational modes of the bubble oscillations.

The purpose of the present paper is to develop a theoretical model for acoustic streaming produced by a cylindrical bubble placed between two elastic solid walls, assuming that the bubble undergoes volume and translational oscillations. An important point to be taken into consideration is that, in the case under study, the bubble generates two types of scattered waves, namely, the bulk scattered wave in the fluid gap and surface waves in the elastic walls, which, in turn, induce Lamb-type surface waves in the fluid channel [33,34]. The previous studies applied a phenomenological model for bubble oscillation [32,35], which is based on qualitative reasoning rather than on a rigorous mathematical derivation and assumes only the existence of the surface waves, while the existence of the bulk wave is ignored. Since the calculation of acoustic streaming is based on the solution given by the bubble oscillation model, the equations of acoustic streaming obtained in Ref. [32] are not mathematically rigorous either. Moreover, they are only applicable to streaming outside the boundary layer. In the present study, the calculation of acoustic streaming is performed using an improved bubble model that was developed in our recent paper [34]. The model is based on rigorous mathematical solutions to the equations of the elastic motion of the channel walls and the equations of the fluid motion in the interior of the channel. It takes into account the existence of both surface and bulk waves. The present study provides solutions for acoustic streaming in the entire space. It should be also emphasized that, in contrast to most studies on acoustic streaming, our derivation does not impose any restriction on the ratio between the viscous penetration depth and the bubble size, so the developed model can be applied to high-viscosity fluids.

\section{THEORY}

The geometry of the problem under consideration is shown in Fig. 1. A cylindrical bubble is squeezed between two planar elastic walls in a microfluidic channel. It should be recognized that real microfluidic bubbles have a pancakelike form [35]. To make the problem amenable to analytical solution, we neglect the curvature of the bubble surface along the $z$ direction and assume that the bubble shape is cylindrical and the scattered acoustic field is predominantly two dimensional. Ilinskii et al. 
(a)

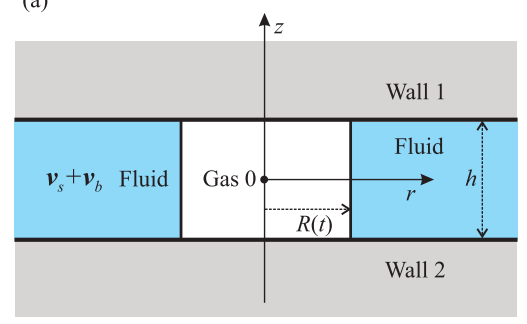

(b)

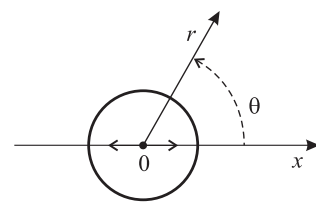

FIG. 1. A cylindrical bubble is squeezed between two planar elastic walls in a microfluidic channel. (a) Side view. (b) Top view.

[36] and others (see references in Ref. [36]) have shown that this approximation works well for bubbles in a narrow channel. This approach also agrees with experimental observations reported in Refs. [16] and [30], which show that the long-range streaming is well captured by a two-dimensional (2D) model.

It is assumed that the bubble undergoes volume and translational oscillations, which will be called modes 0 and 1 , respectively. The volume oscillation is assumed to be caused by an imposed acoustic pressure field and to include two components, one of which is related to the bulk scattered wave in the fluid gap and the second is related to the surface waves propagating in the elastic walls. The translational oscillation is assumed to be induced by the velocity field of an external sound source such as another bubble or an oscillatory fluid flow. Since the problem under consideration is axisymmetric, cylindrical coordinates are used. Their origin is located in the middle of the bubble with the axis $z$ perpendicular to the surface of the walls and the azimuthal angle $\theta$ measured from the direction of the bubble translation; see Fig. 1. It should be emphasized that the mathematical derivation presented below is completely different from that used previously in Ref. [32].

\section{A. Linear solutions}

\section{Linear equations of fluid motion}

The linear velocity field produced by the bubble in the fluid can be represented as

$$
\boldsymbol{v}=\boldsymbol{v}_{s}+\boldsymbol{v}_{b},
$$

where $\boldsymbol{v}_{s}$ is the fluid velocity caused by the surface waves propagating in the walls and $\boldsymbol{v}_{b}$ is the velocity of the bulk scattered wave propagating in the fluid gap. $\boldsymbol{v}_{s}$ can be written as

$$
\boldsymbol{v}_{s}=v_{s}(r) e^{-i \omega t} \boldsymbol{e}_{r},
$$

where $\boldsymbol{e}_{r}$ is the unit radial vector of the cylindrical coordinates and $v_{s}(r)$ is defined by Eq. (2.60) of Ref. [34], in which $z$ is set equal to $0 . \boldsymbol{v}_{b}$ can be represented as

$$
\boldsymbol{v}_{b}=e^{-i \omega t}\left[v_{b r}(r, \theta) \boldsymbol{e}_{r}+v_{b \theta}(r, \theta) \boldsymbol{e}_{\theta}\right],
$$

where $\boldsymbol{e}_{\theta}$ is the unit azimuth vector. If the fluid is treated as incompressible, $\boldsymbol{v}_{b}$ obeys the volume continuity equation,

$$
\nabla \cdot \boldsymbol{v}_{b}=0,
$$

and the incompressible Navier-Stokes equation,

$$
\rho_{f} \frac{\partial \boldsymbol{v}_{b}}{\partial t}-\eta_{f} \Delta \boldsymbol{v}_{b}=-\nabla p_{b}
$$

where $\rho_{f}$ is the fluid density, $\eta_{f}$ is the fluid dynamic viscosity, and $p_{b}$ is the pressure of the bulk wave. Since the time dependence is taken as $\exp (-i \omega t)$, Eq. (5) is transformed to

$$
\left(\Delta+k_{v}^{2}\right) \boldsymbol{v}_{b}=\frac{1}{\eta_{f}} \nabla p_{b},
$$

where

$$
k_{v}=\frac{1+i}{\delta_{v}}, \quad \delta_{v}=\sqrt{\frac{2 v_{f}}{\omega}}, \quad v_{f}=\frac{\eta_{f}}{\rho_{f}},
$$

$v_{f}$ and $\delta_{v}$ being the fluid kinematic viscosity and the viscous penetration depth, respectively.

To satisfy Eq. (4), $\boldsymbol{v}_{b}$ can be expressed in terms of a vector potential $\psi$ as

$$
\boldsymbol{v}_{b}=\nabla \times \boldsymbol{\psi}, \quad \boldsymbol{\psi}=e^{-i \omega t} \psi(r, \theta) \boldsymbol{e}_{z},
$$

where $\boldsymbol{e}_{z}$ is the unit vector along the axis $z$. From Eq. (8) it follows that

$$
v_{b r}=\frac{1}{r} \frac{\partial \psi}{\partial \theta}, \quad v_{b \theta}=-\frac{\partial \psi}{\partial r},
$$

i.e., $\psi$ is the stream function of the bulk wave. Substituting Eq. (8) into Eq. (6) and applying the curl operator, one obtains

$$
\left(\Delta+k_{v}^{2}\right) \Delta \psi=0 .
$$

Considering that the bubble undergoes both radial and translational oscillations, a solution to Eq. (10) can be taken as

$$
\psi=A \theta+B \frac{R_{0}}{r} \sin \theta+C H_{1}^{(1)}\left(k_{v} r\right) \sin \theta,
$$

where $R_{0}$ is the equilibrium bubble radius, $H_{n}^{(1)}$ is the Hankel function of the first kind of order $n$ [37], and $A, B, C$ are constants to be determined from the boundary conditions at the bubble surface. Note that the first two terms on the right-hand side of Eq. (11) are the monopole and the dipole solutions of the equation $\Delta \psi=0$ and the third term is the dipole solution of the equation $\left(\Delta+k_{v}^{2}\right) \psi=0$ [see Eq. (10)]. Thus the term with $A$ describes the contribution of the bubble volume oscillation to the scattered wave and the terms with $B$ and $C$ describe the contribution of the bubble translation, the term with $C$ allowing for the effect of the fluid viscosity.

Substitution of Eq. (11) into Eq. (9) yields

$$
\begin{gathered}
v_{b r}=\frac{A}{r}+\left[B \frac{R_{0}}{r^{2}}+\frac{C}{r} H_{1}^{(1)}\left(k_{v} r\right)\right] \cos \theta, \\
v_{b \theta}=\left(B \frac{R_{0}}{r^{2}}-\frac{C}{r}\left[k_{v} r H_{0}^{(1)}\left(k_{v} r\right)-H_{1}^{(1)}\left(k_{v} r\right)\right]\right) \sin \theta .
\end{gathered}
$$

To find the constants $A, B$, and $C$, three boundary conditions at the bubble surface are used: continuity of normal velocity, zero tangential stress, and normal stress balance. These conditions are considered in the next subsection.

\section{Boundary conditions for linear solutions}

The total fluid velocity, in addition to $\boldsymbol{v}_{s}$ and $\boldsymbol{v}_{b}$, includes a velocity produced by a source of the bubble translation, such as another bubble. Let us denote this velocity by $\boldsymbol{v}_{e x}$. Since we assume that the bubble translates along the axis $x$ (see Fig. 1), 
$\boldsymbol{v}_{e x}$ should have the same direction and therefore can be taken as

$$
\boldsymbol{v}_{e x}=v_{e x} e^{-i \omega t} \boldsymbol{e}_{x}=v_{e x} e^{-i \omega t}\left(\boldsymbol{e}_{r} \cos \theta-\boldsymbol{e}_{\theta} \sin \theta\right),
$$

where $\boldsymbol{e}_{x}$ is the unit vector along the axis $x$. Taking into account Eqs. (2), (3), and (14), the boundary condition for normal velocity at the bubble surface is written as

$$
\left(v_{s}+v_{b r}+v_{e x} \cos \theta\right) e^{-i \omega t}=\frac{d R}{d t}+\frac{d \boldsymbol{x}}{d t} \cdot \boldsymbol{e}_{r} \quad \text { at } \quad r=R_{0},
$$

where $R(t)$ is the time-varying bubble radius and $\boldsymbol{x}(t)$ is the translational displacement of the bubble center. These quantities can be defined by

$$
R(t)=R_{0}\left(1+a e^{-i \omega t}\right), \quad \boldsymbol{x}(t)=x_{m} e^{-i \omega t} \boldsymbol{e}_{x} .
$$

Substituting Eqs. (12) and (16) into Eq. (15) and separating terms without and with $\cos \theta$, one obtains

$$
\begin{gathered}
A=-i \omega R_{0}^{2} a-R_{0} v_{s}\left(R_{0}\right), \\
x_{m}=\frac{i}{\omega R_{0}}\left[R_{0} v_{e x}+B+C H_{1}^{(1)}\left(\alpha_{v}\right)\right],
\end{gathered}
$$

where $\alpha_{v}=k_{v} R_{0}$.

We assume that the bubble surface is free of impurities and surfactants and, as the viscosity of the gas inside the bubble is much smaller than that of the fluid, slippage on the gas-fluid interface occurs. Then we can apply the boundary condition of zero tangential stress, which is given by

$$
\sigma_{r \theta}=0 \quad \text { at } \quad r=R_{0},
$$

where the tangential stress $\sigma_{r \theta}$ is calculated by [38]

$$
\sigma_{r \theta}=\eta_{f}\left(\frac{1}{r} \frac{\partial v_{b r}}{\partial \theta}+\frac{\partial v_{b \theta}}{\partial r}-\frac{v_{b \theta}}{r}\right) e^{-i \omega t} .
$$

Substitution of Eqs. (12) and (13) into Eq. (20) results in

$$
\begin{aligned}
\sigma_{r \theta}= & -\frac{\eta_{f} \sin \theta}{r^{2}}\left(\frac{4 R_{0}}{r} B+C\left[\left(4-k_{v}^{2} r^{2}\right) H_{1}^{(1)}\left(k_{v} r\right)\right.\right. \\
& \left.\left.-2 k_{v} r H_{0}^{(1)}\left(k_{v} r\right)\right]\right) e^{-i \omega t} .
\end{aligned}
$$

On substitution of Eq. (21) into Eq. (19), one obtains

$$
C=\frac{4 B}{2 \alpha_{v} H_{0}^{(1)}\left(\alpha_{v}\right)+\left(\alpha_{v}^{2}-4\right) H_{1}^{(1)}\left(\alpha_{v}\right)} .
$$
by

The boundary condition of normal stress balance is given

$$
p_{g}=P_{0}+p_{a c}+p_{s t}+p_{s}+p_{e x}-\sigma_{r r} \quad \text { at } \quad r=R_{0},
$$

where $p_{g}$ is the gas pressure in the bubble, $P_{0}$ is the hydrostatic pressure in the fluid, $p_{a c}=P_{a} \exp (-i \omega t)$ is the driving acoustic pressure, where $P_{a}$ stands for the pressure amplitude, $p_{s t}$ is the surface tension pressure, $p_{s}$ is the fluid pressure caused by the surface waves [it is given by Eq. (2.62) of Ref. [34]], $p_{e x}$ is the fluid pressure corresponding to the velocity $\boldsymbol{v}_{e x}$, and $\sigma_{r r}$ is the normal stress produced by the bulk wave. The terms of Eq. (23) are calculated by equations that follow.
With the bubble volume given by

$$
V=\pi R^{2} h \approx \pi R_{0}^{2} h\left(1+2 a e^{-i \omega t}\right)=V_{0}\left(1+2 a e^{-i \omega t}\right),
$$

where $h$ is the height of the fluid channel, $p_{g}$ is calculated by

$$
p_{g}=P_{g 0}\left(\frac{V_{0}}{V}\right)^{\gamma} \approx P_{g 0}\left(1-2 \gamma a e^{-i \omega t}\right),
$$

where $P_{g 0}$ is the equilibrium gas pressure and $\gamma$ is the ratio of specific heats of the gas. For $p_{s t}$, one has

$$
p_{s t}=\frac{\sigma_{f}}{R} \approx \frac{\sigma_{f}}{R_{0}}\left(1-a e^{-i \omega t}\right),
$$

where $\sigma_{f}$ is the surface tension coefficient for the fluid-gas interface. The value of $p_{e x}$ at $r=R_{0}$ is given by

$$
\begin{aligned}
\left.p_{e x}\right|_{r=R_{0}} & =\left.p_{e x}\right|_{r=0}+R_{0} \boldsymbol{e}_{r} \cdot \nabla p_{e x} \\
& =\left(p_{e x 0}+i \omega \rho_{f} R_{0} v_{e x} \cos \theta\right) e^{-i \omega t},
\end{aligned}
$$

where $p_{e x 0}$ is the amplitude of $p_{e x}$ at the center of the bubble and the expression on the right-hand side of Eq. (27) was obtained by the following relation:

$$
\nabla p_{e x}=-\rho_{f} \frac{\partial \boldsymbol{v}_{e x}}{\partial t}=i \omega \rho_{f} \boldsymbol{v}_{e x} .
$$

Equation (27) provides the contribution of $p_{e x}$ to the normal stress balance with the same accuracy as the contribution of $\boldsymbol{v}_{e x}$ to the boundary condition for normal velocity.

It remains to calculate $\sigma_{r r}$, which is given by [38]

$$
\sigma_{r r}=-p_{b}+2 \eta_{f} \frac{\partial v_{b r}}{\partial r} e^{-i \omega t}
$$

To find $p_{b}$, Eq. (6) is used. Substitution of Eq. (8) into the left-hand side of Eq. (6) yields

$$
\begin{aligned}
\left(\Delta+k_{v}^{2}\right) \boldsymbol{v}_{b} & =\left(\Delta+k_{v}^{2}\right)(\nabla \times \boldsymbol{\psi})=e^{-i \omega t} \nabla \times\left[\boldsymbol{e}_{z}\left(\Delta+k_{v}^{2}\right) \psi\right] \\
& =e^{-i \omega t} k_{v}^{2} \nabla \times\left[\boldsymbol{e}_{z}\left(A \theta+B \frac{R_{0}}{r} \sin \theta\right)\right] .
\end{aligned}
$$

It is easy to check by direct calculation that the last expression on the right-hand side of Eq. (30) is identical to

$$
e^{-i \omega t} k_{v}^{2} \nabla\left(A \ln (r)-B \frac{R_{0}}{r} \cos \theta\right) .
$$

Substituting Eq. (31) into Eq. (6), one obtains

$$
p_{b}=i \omega \rho_{f} e^{-i \omega t}\left(A \ln (r)-B \frac{R_{0}}{r} \cos \theta+\mathrm{const}\right) \text {. }
$$

Equation (32) shows a well-known problem that arises in an incompressible fluid, namely, the pressure generated by the bubble does not vanish as $r \rightarrow \infty$. The conventional solution of this problem consists of limiting the radial extent of an incompressible fluid to a finite distance $r_{\max }$ [36]. To this end, the arbitrary constant in Eq. (32) is set equal to $-A \ln \left(r_{\max }\right)$ so that Eq. (32) takes the form

$$
p_{b}=-i \omega \rho_{f} e^{-i \omega t}\left[A \ln \left(\frac{r_{\max }}{r}\right)+B \frac{R_{0}}{r} \cos \theta\right] \text {. }
$$

In our study, this point is of no importance because $p_{b}$ is not used in calculating acoustic streaming. Equation (33) is required only in order to obtain a relationship between the 
constants $B$ and $C$ from the normal stress balance. We will see below that this aim does not require the use of the logarithmic term of Eq. (33).

Substitution of Eqs. (12) and (33) into Eq. (29) gives $\sigma_{r r}$. Substituting $\sigma_{r r}$ along with the other quantities calculated above into Eq. (23) and separating out terms with $\cos \theta$, one obtains

$$
\left(\alpha_{v}^{2}-4\right) B+2\left[\alpha_{v} H_{0}^{(1)}\left(\alpha_{v}\right)-2 H_{1}^{(1)}\left(\alpha_{v}\right)\right] C=\alpha_{v}^{2} R_{0} v_{e x} .
$$

In combination with Eq. (22), Eq. (34) gives

$$
\begin{gathered}
B=R_{0} v_{e x} \frac{2 \alpha_{v} H_{0}^{(1)}\left(\alpha_{v}\right)+\left(\alpha_{v}^{2}-4\right) H_{1}^{(1)}\left(\alpha_{v}\right)}{2 \alpha_{v} H_{0}^{(1)}\left(\alpha_{v}\right)+\left(\alpha_{v}^{2}-8\right) H_{1}^{(1)}\left(\alpha_{v}\right)}, \\
C=\frac{4 R_{0} v_{e x}}{2 \alpha_{v} H_{0}^{(1)}\left(\alpha_{v}\right)+\left(\alpha_{v}^{2}-8\right) H_{1}^{(1)}\left(\alpha_{v}\right)} .
\end{gathered}
$$

Note that the logarithmic term of Eq. (33) is not involved in these calculations. Substituting Eqs. (35) and (36) into Eq. (18), one obtains the amplitude of the bubble translational displacement:

$$
x_{m}=\frac{2 i B}{\omega R_{0}}=\frac{2 i v_{e x}}{\omega}\left(\frac{2 \alpha_{v} H_{0}^{(1)}\left(\alpha_{v}\right)+\left(\alpha_{v}^{2}-4\right) H_{1}^{(1)}\left(\alpha_{v}\right)}{2 \alpha_{v} H_{0}^{(1)}\left(\alpha_{v}\right)+\left(\alpha_{v}^{2}-8\right) H_{1}^{(1)}\left(\alpha_{v}\right)}\right) \text {. }
$$

It should be mentioned that the terms of Eq. (23) without $\cos \theta$ give an equation for the amplitude of the bubble pulsation $a$. This equation reduces to the well-known equation for the pulsation of a cylindrical bubble in an incompressible fluid if terms related to the surface waves are omitted; see, e.g., Ref. [36]. We do not consider this equation because it is more reasonable to use the equation for $a$ derived in Ref. [34], which takes the fluid compressibility into account and hence is free from problems related to the logarithmic term in Eq. (33).

\section{B. Solutions of the equations of acoustic streaming}

\section{Method of solution}

The equations of acoustic streaming are given by [4]

$$
\begin{gathered}
\nabla \cdot \boldsymbol{V}=0, \\
v_{f} \Delta \boldsymbol{V}-\frac{1}{\rho_{f}} \nabla P=\langle(\boldsymbol{v} \cdot \nabla) \boldsymbol{v}\rangle,
\end{gathered}
$$

where $\boldsymbol{V}$ and $P$ are the Eulerian streaming velocity and pressure fields, \langle\rangle means time averaging, and $\boldsymbol{v}$ is the linear fluid velocity. To satisfy Eq. (38), $\boldsymbol{V}$ is taken as

$$
\boldsymbol{V}=\boldsymbol{\nabla} \times \boldsymbol{\Psi} .
$$

Substituting Eq. (40) into Eq. (39) and applying the curl operator, one obtains

$$
\Delta^{2} \boldsymbol{\Psi}=-\frac{1}{v_{f}} \nabla \times \boldsymbol{W},
$$

where

$$
\boldsymbol{W}=\langle(\boldsymbol{v} \cdot \nabla) \boldsymbol{v}\rangle
$$

This nonlinear quantity results from the convection term of the nonlinear Navier-Stokes equation.

In our case, $\boldsymbol{\Psi}$ can be written as

$$
\Psi=\Psi(r, \theta) e_{z} .
$$

Substitution of Eq. (43) into Eq. (40) shows that the radial and azimuthal components of $\boldsymbol{V}$ are given by

$$
V_{r}=\frac{1}{r} \frac{\partial \Psi}{\partial \theta}, \quad V_{\theta}=-\frac{\partial \Psi}{\partial r},
$$

which means that $\Psi$ can be treated as the stream function of acoustic streaming. Substituting Eq. (43) into Eq. (41), one obtains

$$
\Delta^{2} \Psi=-\frac{1}{v_{f} r}\left[\frac{\partial\left(r W_{\theta}\right)}{\partial r}-\frac{\partial W_{r}}{\partial \theta}\right] .
$$

We will see below that for streaming produced by modes 0 (volume oscillation) and 1 (translation), the function on the right-hand side of Eq. (45) depends on $\theta$ as $\sin \theta$. For modes 1 and 1 or 0 and 2 (quadrupole oscillation), the dependence on $\theta$ is given by $\sin 2 \theta[29,30]$. Thus, for two arbitrary modes, Eq. (45) can be represented as

$$
\Delta^{2} \Psi=F(r) \sin n \theta,
$$

where $n=1,2 \ldots$ and $F(r)$ is calculated by substituting corresponding linear solutions into Eq. (42).

Equation (46) suggests that $\Psi$ should be sought in the following form:

$$
\Psi=G(r) \sin n \theta .
$$

Substitution of Eq. (47) into Eq. (46) yields

$$
\begin{aligned}
\frac{d^{4} G}{d r^{4}} & +\frac{2}{r} \frac{d^{3} G}{d r^{3}}-\frac{2 n^{2}+1}{r^{2}} \frac{d^{2} G}{d r^{2}}+\frac{2 n^{2}+1}{r^{3}} \frac{d G}{d r} \\
& +\frac{n^{2}\left(n^{2}-4\right)}{r^{4}} G=F .
\end{aligned}
$$

In the theory of ordinary differential equations (ODE), Eq. (48) is called the nonhomogeneous Cauchy-Euler equation. It can be solved by the so-called method of variation of parameters, also known as the Lagrange method [39]. According to this method, the homogeneous equation corresponding to Eq. (48) is first solved,

$$
\begin{aligned}
\frac{d^{4} G}{d r^{4}} & +\frac{2}{r} \frac{d^{3} G}{d r^{3}}-\frac{2 n^{2}+1}{r^{2}} \frac{d^{2} G}{d r^{2}}+\frac{2 n^{2}+1}{r^{3}} \frac{d G}{d r} \\
& +\frac{n^{2}\left(n^{2}-4\right)}{r^{4}} G=0 .
\end{aligned}
$$

Solutions to Eq. (49) are sought as $r^{\lambda}$. Substitution of this expression into Eq. (49) yields

$$
\begin{gathered}
\lambda(\lambda-1)(\lambda-2)(\lambda-3)+2 \lambda(\lambda-1)(\lambda-2) \\
-\left(2 n^{2}+1\right) \lambda(\lambda-2)+n^{2}\left(n^{2}-4\right)=0 .
\end{gathered}
$$

This equation is the characteristic equation of the differential equation (49). Its roots determine the partial solutions of Eq. (49). Equation (50) is of fourth order in $\lambda$. Hence it has four roots and if all of them are different, the fundamental system of solutions of Eq. (49) is given by

$$
s_{1}=r^{\lambda_{1}}, \quad s_{2}=r^{\lambda_{2}}, \quad s_{3}=r^{\lambda_{3}}, \quad s_{4}=r^{\lambda_{4}} .
$$


If there are multiple (identical) roots, for example, $\lambda_{1}=\lambda_{2}$, the fundamental solutions are given by

$$
s_{1}=r^{\lambda_{1}}, \quad s_{2}=r^{\lambda_{1}} \ln (r), \quad s_{3}=r^{\lambda_{3}}, \quad s_{4}=r^{\lambda_{4}} .
$$

The general solution of Eq. (49) is the sum of the fundamental solutions with arbitrary constants,

$$
G=G_{1} s_{1}+G_{2} s_{2}+G_{3} s_{3}+G_{4} s_{4} .
$$

In order to find the solution of Eq. (48), the constants $G_{n}$ in Eq. (53) are replaced with functions $G_{n}(r)$,

$$
G=G_{1}(r) s_{1}+G_{2}(r) s_{2}+G_{3}(r) s_{3}+G_{4}(r) s_{4} .
$$

According to the theory of ODE, Eq. (54) provides the solution of Eq. (48) if the functions $G_{n}(r)$ obey the following system of equations:

$$
\begin{aligned}
G_{1}^{\prime} s_{1}+G_{2}^{\prime} s_{2}+G_{3}^{\prime} s_{3}+G_{4}^{\prime} s_{4} & =0, \\
G_{1}^{\prime} s_{1}^{\prime}+G_{2}^{\prime} s_{2}^{\prime}+G_{3}^{\prime} s_{3}^{\prime}+G_{4}^{\prime} s_{4}^{\prime} & =0, \\
G_{1}^{\prime} s_{1}^{\prime \prime}+G_{2}^{\prime} s_{2}^{\prime \prime}+G_{3}^{\prime} s_{3}^{\prime \prime}+G_{4}^{\prime} s_{4}^{\prime \prime} & =0, \\
G_{1}^{\prime} s_{1}^{\prime \prime \prime}+G_{2}^{\prime} s_{2}^{\prime \prime \prime}+G_{3}^{\prime} s_{3}^{\prime \prime \prime}+G_{4}^{\prime} s_{4}^{\prime \prime \prime} & =F(r),
\end{aligned}
$$

where the prime denotes the derivative over $r$. From this system, we can find expressions for $G_{n}^{\prime}$ and then integrate them. As a result, we will obtain expressions for $G_{n}(r)$ with four arbitrary constants $G_{n 0}$. The constants are calculated from boundary conditions at infinity and at the bubble surface.

\section{Calculation of the function $F(r)$ for modes 0 and 1}

Let us split the linear velocity $v$ into modes 0 and 1 by introducing the following designations:

$$
\begin{gathered}
\boldsymbol{v}^{(0)}=e^{-i \omega t}\left(v_{s}+v_{b r}^{(0)}\right) \boldsymbol{e}_{r}, \\
\boldsymbol{v}^{(1)}=e^{-i \omega t}\left(v_{b r}^{(1)} \boldsymbol{e}_{r}+v_{b \theta}^{(1)} \boldsymbol{e}_{\theta}\right),
\end{gathered}
$$

where the superscripts (0) and (1) denote quantities related to modes 0 and 1, respectively. From Eqs. (12) and (13) it follows that

$$
\begin{gathered}
v_{b r}^{(0)}=\frac{A}{r}, \\
v_{b r}^{(1)}=\left(B \frac{R_{0}}{r^{2}}+\frac{C}{r} H_{1}^{(1)}\left(k_{v} r\right)\right) \cos \theta, \\
v_{b \theta}^{(1)}=\left(B \frac{R_{0}}{r^{2}}-\frac{C}{r}\left[k_{v} r H_{0}^{(1)}\left(k_{v} r\right)-H_{1}^{(1)}\left(k_{v} r\right)\right]\right) \sin \theta .
\end{gathered}
$$

Substituting Eqs. (56) and (57) into Eq. (42) and keeping only the mixed-mode terms, one obtains

$$
\begin{aligned}
\boldsymbol{W}^{(01)}= & \left\langle\left(\boldsymbol{v}^{(0)} \cdot \nabla\right) \boldsymbol{v}^{(1)}+\left(\boldsymbol{v}^{(1)} \cdot \nabla\right) \boldsymbol{v}^{(0)}\right\rangle \\
= & \frac{1}{2} \operatorname{Re}\left[\left(v_{s}+v_{b r}^{(0)}\right)^{*} \frac{\partial}{\partial r}\left(v_{b r}^{(1)} \boldsymbol{e}_{r}+v_{b \theta}^{(1)} \boldsymbol{e}_{\theta}\right)\right. \\
& \left.+\left(v_{b r}^{(1)} \frac{\partial}{\partial r}+\frac{v_{b \theta}^{(1)}}{r} \frac{\partial}{\partial \theta}\right)\left[\boldsymbol{e}_{r}\left(v_{s}+v_{b r}^{(0)}\right)^{*}\right]\right],
\end{aligned}
$$

where the asterisk denotes the complex conjugate. From Eq. (61), one finds the $r$ and $\theta$ components of $\boldsymbol{W}^{(01)}$ to be

$$
\begin{aligned}
W_{r}^{(01)} & =\frac{1}{2} \operatorname{Re}\left(\left(v_{s}+v_{b r}^{(0)}\right)^{*} \frac{\partial v_{b r}^{(1)}}{\partial r}+v_{b r}^{(1)} \frac{\partial}{\partial r}\left(v_{s}+v_{b r}^{(0)}\right)^{*}\right) \\
& =\frac{1}{2} \operatorname{Re}\left(\frac{\partial}{\partial r}\left[v_{b r}^{(1)}\left(v_{s}+v_{b r}^{(0)}\right)^{*}\right]\right), \\
W_{\theta}^{(01)} & =\frac{1}{2} \operatorname{Re}\left(\left(v_{s}+v_{b r}^{(0)}\right)^{*} \frac{\partial v_{b \theta}^{(1)}}{\partial r}+\frac{v_{b \theta}^{(1)}}{r}\left(v_{s}+v_{b r}^{(0)}\right)^{*}\right) \\
& =\frac{1}{2} \operatorname{Re}\left(\left(v_{s}+v_{b r}^{(0)}\right)^{*} \frac{1}{r} \frac{\partial\left(r v_{b \theta}^{(1)}\right)}{\partial r}\right) .
\end{aligned}
$$

Note that in Eq. (63) the relation $\boldsymbol{e}_{\theta}=\partial \boldsymbol{e}_{r} / \partial \theta$ was used. Substitution of Eqs. (62) and (63) into the right-hand side of Eq. (45) leads to

$$
\begin{aligned}
& -\frac{1}{v_{f} r}\left(\frac{\partial\left(r W_{\theta}\right)}{\partial r}-\frac{\partial W_{r}}{\partial \theta}\right) \\
& =-\frac{1}{2 v_{f} r} \operatorname{Re}\left\{\frac{\partial}{\partial r}\left[\left(v_{s}+v_{b r}^{(0)}\right)^{*}\left(\frac{\partial\left(r v_{b \theta}^{(1)}\right)}{\partial r}-\frac{\partial v_{b r}^{(1)}}{\partial \theta}\right)\right]\right\} .
\end{aligned}
$$

Substituting Eqs. (58)-(60) into Eq. (64), one has

$$
\begin{aligned}
- & \frac{1}{v_{f} r}\left(\frac{\partial\left(r W_{\theta}\right)}{\partial r}-\frac{\partial W_{r}}{\partial \theta}\right) \\
=- & \frac{\sin \theta}{2 v_{f} r} \operatorname{Re}\left(k_{v}^{2} C \frac{d}{d r}\left\{H_{1}^{(1)}\left(k_{v} r\right)\left[r v_{s}^{*}(r)+A^{*}\right]\right\}\right) .
\end{aligned}
$$

Comparison with Eq. (46) finally yields

$$
F(r)=-\frac{\omega}{2 v_{f}^{2} r} \operatorname{Re}\left(i C \frac{d}{d r}\left\{H_{1}^{(1)}\left(k_{v} r\right)\left[r v_{s}^{*}(r)+A^{*}\right]\right\}\right) .
$$

Note that at a distance from the bubble, $v_{s}(r)$ can be approximated by a Hankel function. However, in the vicinity of the bubble, $v_{s}(r)$ should be calculated from the integral expression (2.60) in Ref. [34].

\section{Calculation of the function $G(r)$ for modes 0 and 1}

For modes 0 and 1, $n=1$ in Eq. (47). In this case, Eq. (50) takes the form

$$
(\lambda-1)^{2}\left(\lambda^{2}-2 \lambda-3\right)=0 .
$$

The roots of this equation are

$$
\lambda_{1}=\lambda_{2}=1, \quad \lambda_{3}=3, \quad \lambda_{4}=-1 .
$$


With these roots, Eqs. (54) and (55) give

$$
\begin{aligned}
G=G_{1}(r) r+G_{2}(r) r \ln (r)+G_{3}(r) r^{3} & +\frac{G_{4}(r)}{r}, \\
r G_{1}^{\prime}+r \ln (r) G_{2}^{\prime}+r^{3} G_{3}^{\prime}+r^{-1} G_{4}^{\prime} & =0, \\
G_{1}^{\prime}+[1+\ln (r)] G_{2}^{\prime}+3 r^{2} G_{3}^{\prime}-r^{-2} G_{4}^{\prime} & =0, \\
r^{-1} G_{2}^{\prime}+6 r G_{3}^{\prime}+2 r^{-3} G_{4}^{\prime} & =0, \\
-r^{-2} G_{2}^{\prime}+6 G_{3}^{\prime}-6 r^{-4} G_{4}^{\prime} & =F(r) .
\end{aligned}
$$

Solving Eq. (70) yields

$G_{1}^{\prime}=\frac{r^{2} \ln (r) F}{4}, G_{2}^{\prime}=-\frac{r^{2} F}{4}, G_{3}^{\prime}=\frac{F}{16}, G_{4}^{\prime}=-\frac{r^{4} F}{16}$.

After integrating Eq. (71), one has

$$
\begin{gathered}
G_{1}(r)=G_{10}+\frac{1}{4} \int_{R_{0}}^{r} x^{2} \ln (x) F(x) d x, \\
G_{2}(r)=G_{20}-\frac{1}{4} \int_{R_{0}}^{r} x^{2} F(x) d x, \\
G_{3}(r)=G_{30}+\frac{1}{16} \int_{R_{0}}^{r} F(x) d x, \\
G_{4}(r)=G_{40}-\frac{1}{16} \int_{R_{0}}^{r} x^{4} F(x) d x,
\end{gathered}
$$

where $G_{m 0}$ are arbitrary constants.

\section{Expressions for the components of the Eulerian streaming velocity}

Substitution of Eq. (47) into Eq. (44) yields

$$
V_{r}=\frac{G(r)}{r} \cos \theta, \quad V_{\theta}=-G^{\prime}(r) \sin \theta .
$$

From Eqs. (69) and (70) it follows that

$$
G^{\prime}(r)=G_{1}(r)+G_{2}(r)[1+\ln (r)]+3 r^{2} G_{3}(r)-\frac{G_{4}(r)}{r^{2}} .
$$

The unknown constants $G_{m 0}$ appearing in these equations will be calculated by applying the boundary conditions to the Lagrangian streaming velocity, which is calculated in the next subsection.

\section{Lagrangian streaming velocity}

The Lagrangian streaming velocity is given by

$$
\boldsymbol{V}_{L}=\boldsymbol{V}+\boldsymbol{V}_{S},
$$

where $\boldsymbol{V}_{S}$ is the Stokes drift velocity, which is defined by [24]

$$
\boldsymbol{V}_{S}=\left\langle\int \boldsymbol{v} d t \cdot \nabla \boldsymbol{v}\right\rangle
$$

Equation (77) can be transformed as follows:

$$
\begin{aligned}
\boldsymbol{V}_{S}= & \frac{1}{\omega}\langle(i \boldsymbol{v} \cdot \nabla) \boldsymbol{v}\rangle=\frac{1}{\omega}\left\langle\left(i \boldsymbol{v}^{(0)} \cdot \nabla\right) \boldsymbol{v}^{(1)}+\left(i \boldsymbol{v}^{(1)} \cdot \nabla\right) \boldsymbol{v}^{(0)}\right\rangle \\
= & \frac{1}{2 \omega} \operatorname{Re}\left[-i\left(v_{s}+v_{b r}^{(0)}\right)^{*} \frac{\partial}{\partial r}\left(v_{b r}^{(1)} \boldsymbol{e}_{r}+v_{b \theta}^{(1)} \boldsymbol{e}_{\theta}\right)\right. \\
& \left.+i\left(v_{b r}^{(1)} \frac{\partial}{\partial r}+\frac{v_{b \theta}^{(1)}}{r} \frac{\partial}{\partial \theta}\right)\left[\boldsymbol{e}_{r}\left(v_{s}+v_{b r}^{(0)}\right)^{*}\right]\right] .
\end{aligned}
$$

The components of $\boldsymbol{V}_{S}$ are found from Eq. (78) as

$$
V_{S r}=\frac{1}{2 \omega} \operatorname{Re}\left(-i\left(v_{s}+v_{b r}^{(0)}\right)^{*} \frac{\partial v_{b r}^{(1)}}{\partial r}+i v_{b r}^{(1)} \frac{\partial}{\partial r}\left(v_{s}+v_{b r}^{(0)}\right)^{*}\right),
$$

$$
V_{S \theta}=\frac{1}{2 \omega} \operatorname{Re}\left[i\left(v_{s}+v_{b r}^{(0)}\right)^{*}\left(\frac{v_{b \theta}^{(1)}}{r}-\frac{\partial v_{b \theta}^{(1)}}{\partial r}\right)\right] .
$$

On substitution of Eqs. (58)-(60) into Eqs. (79) and (80), one obtains

$$
V_{S r}=S_{r}(r) \cos \theta, \quad V_{S \theta}=S_{\theta}(r) \sin \theta,
$$

where

$$
\begin{aligned}
S_{r}(r)= & \frac{1}{2 \omega r^{3}} \operatorname{Re}\left[i\left(r v_{s}^{*}+A^{*}\right)\right. \\
& \times\left(2 B \frac{R_{0}}{r}-C\left[k_{v} r H_{0}^{(1)}\left(k_{v} r\right)-2 H_{1}^{(1)}\left(k_{v} r\right)\right]\right) \\
& \left.+i\left(r^{2} v_{s}^{\prime *}-A^{*}\right)\left(B \frac{R_{0}}{r}+C H_{1}^{(1)}\left(k_{v} r\right)\right)\right], \\
S_{\theta}(r)= & \frac{1}{2 \omega r^{3}} \operatorname{Re}\left[i ( r v _ { s } ^ { * } + A ^ { * } ) \left(3 B \frac{R_{0}}{r}\right.\right. \\
& \left.\left.+C\left[\left(3-k_{v}^{2} r^{2}\right) H_{1}^{(1)}\left(k_{v} r\right)-2 k_{v} r H_{0}^{(1)}\left(k_{v} r\right)\right]\right)\right] .
\end{aligned}
$$

Thus, the components of $\boldsymbol{V}_{L}$ are given by

$$
V_{L r}=U_{r}(r) \cos \theta, \quad V_{L \theta}=U_{\theta}(r) \sin \theta,
$$

where

$$
U_{r}(r)=S_{r}(r)+\frac{G(r)}{r}, \quad U_{\theta}(r)=S_{\theta}(r)-G^{\prime}(r) .
$$

\section{Calculation of the constants $\boldsymbol{G}_{m 0}$} that

The condition of zero streaming velocity at infinity requires

$$
\begin{aligned}
G_{10} & =-\frac{1}{4} \int_{R_{0}}^{\infty} x^{2} \ln (x) F(x) d x, \\
G_{20} & =\frac{1}{4} \int_{R_{0}}^{\infty} x^{2} F(x) d x, \\
G_{30} & =-\frac{1}{16} \int_{R_{0}}^{\infty} F(x) d x .
\end{aligned}
$$

These equations follow from Eqs. (72) and (73). 
To find $G_{40}$, the condition of continuity of normal velocity at the bubble surface is applied. It is shown in Ref. [30] that in terms of Lagrangian streaming, the above condition can be written as

$$
V_{L r}=0 \quad \text { at } \quad r=R_{0} .
$$

Substitution of the first of Eqs. (84) into Eq. (87) yields

$$
G_{40}=-R_{0}^{2}\left[S_{r}\left(R_{0}\right)+G_{10}+\ln \left(R_{0}\right) G_{20}+R_{0}^{2} G_{30}\right] .
$$

\section{Limit of low viscosity}

In most studies on acoustic streaming, solutions are calculated in the limit of low viscosity, assuming that $\delta_{v} / R_{0} \ll 1$. In our case, this condition is valid as well. Therefore, it is appropriate to consider this limit and to simplify the equations derived above.

For $\delta_{v} / R_{0} \ll 1$, Eqs. (35) and (36) reduce to

$$
B=R_{0} v_{e x}, C=2(i-1) \sqrt{\pi} R_{0} \alpha_{v}^{-3 / 2} e^{-i \alpha_{v}} v_{e x} .
$$

Recall that the constants $B$ and $C$ are related to the bubble translation; see the paragraph following Eq. (11). Substitution of $B$ from Eq. (89) into Eq. (37) yields

$$
x_{m 0}=2 i v_{e x} / \omega, \quad u_{m 0}=-i \omega x_{m 0}=2 v_{e x},
$$

where $x_{m 0}$ and $u_{m 0}$ denote, respectively, the amplitude of the bubble translational displacement and the amplitude of the bubble translational velocity at $\delta_{v} / R_{0} \ll 1$. Note that $u_{m 0}$ is twice the fluid velocity that forces the bubble into the translational motion. For a spherical bubble, this factor is 3 [38].

In the limit $\delta_{v} / R_{0} \ll 1$, Eq. (85) take the form

$$
\begin{gathered}
U_{r}(r)=\frac{1}{4 r^{2}} \operatorname{Re}\left[x _ { m 0 } ^ { * } \left(\frac{R_{0}^{2}}{r^{2}}\left[A+2 r v_{s}(r)+r^{2} v_{s}^{\prime}(r)\right]\right.\right. \\
\left.\left.-A-2 R_{0} v_{s}\left(R_{0}\right)-R_{0}^{2} v_{s}^{\prime}\left(R_{0}\right)\right)\right], \\
U_{\theta}(r)=\frac{1}{4 r^{2}} \operatorname{Re}\left[x _ { m 0 } ^ { * } \left(\frac{3 R_{0}^{2}}{r^{2}}\left[A+r v_{s}(r)\right]\right.\right. \\
\left.\left.-A-2 R_{0} v_{s}\left(R_{0}\right)-R_{0}^{2} v_{s}^{\prime}\left(R_{0}\right)\right)\right] .
\end{gathered}
$$

If there are no surface waves $\left(v_{s}=0\right)$, Eqs. (91) and (92) reduce to

$$
\begin{aligned}
U_{r}(r) & =\frac{1}{4} \omega|a|\left|x_{m 0}\right| \sin (\Delta \phi)\left(\frac{R_{0}^{2}}{r^{2}}-\frac{R_{0}^{4}}{r^{4}}\right), \\
U_{\theta}(r) & =\frac{1}{4} \omega|a|\left|x_{m 0}\right| \sin (\Delta \phi)\left(\frac{R_{0}^{2}}{r^{2}}-\frac{3 R_{0}^{4}}{r^{4}}\right),
\end{aligned}
$$

where $\Delta \phi=\phi_{1}-\phi_{0}$ with $\phi_{0}$ and $\phi_{1}$ being the arguments of $a$ and $x_{m 0}$. These equations agree with the results of MekkiBerrada et al. [32].

Equations (93) and (94) show that the velocity components are proportional to $\sin (\Delta \phi)$. For $v_{s} \neq 0$, the dependence on $\Delta \phi$ is not so simple. The complexity of the equations with $v_{s} \neq 0$ makes it impossible to see straight away the dependence of
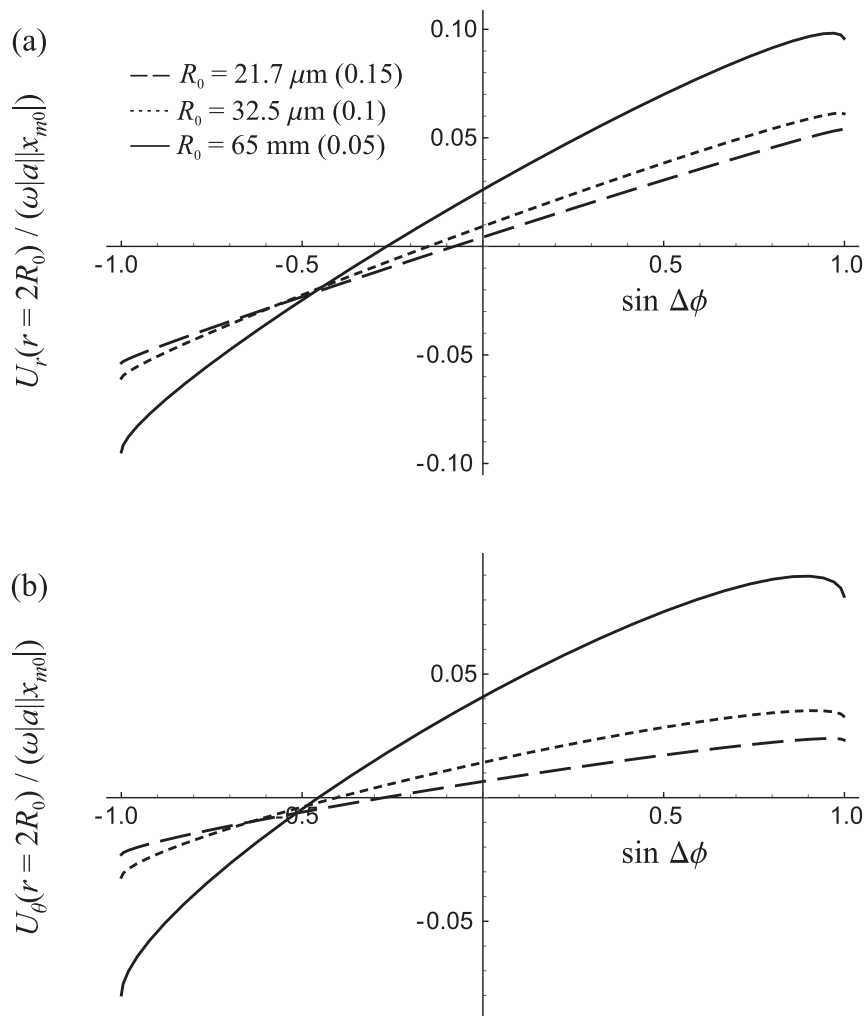

FIG. 2. Dependence of the streaming velocity on the phase shift $\Delta \phi$ between the radial and translational oscillations. Normalized (a) radial and (b) azimuthal velocity components vs $\sin (\Delta \phi)$ for three values of $R_{0}$. The numbers in brackets are the values of the ratio $\delta_{v} / R_{0}$.

$U_{r}$ and $U_{\theta}$ on $\Delta \phi$. Therefore, we have to resort to numerical examples. Figure 2 shows the values of $U_{r}$ and $U_{\theta}$, calculated at $r / R_{0}=2$ and normalized by $\omega|a|\left|x_{m 0}\right|$, versus $\sin (\Delta \phi)$. The simulations were made at the driving frequency $f=30 \mathrm{kHz}$ for three values of $R_{0}: 21.7 \mu \mathrm{m}\left(\delta_{v} / R_{0}=0.15\right), 32.5 \mu \mathrm{m}$ $\left(\delta_{v} / R_{0}=0.1\right)$, and $65 \mu \mathrm{m}\left(\delta_{v} / R_{0}=0.05\right)$. Figure 2 reveals that for $v_{s} \neq 0$, the streaming velocity is not linearly dependent on $\sin (\Delta \phi)$ and does not vanish at $\Delta \phi=0$.

\section{Calculation of $v_{e x}, p_{e x}$, and $a$ for the case of two bubbles}

Let us assume that the translational motion of the bubble under consideration (bubble 1) is induced by a neighboring bubble (bubble 2) located at a distance $d$ as shown in Fig. 3 . It is evident that not only does bubble 2 affect the oscillation of bubble 1 , but bubble 1 affects the oscillation of bubble 2 as

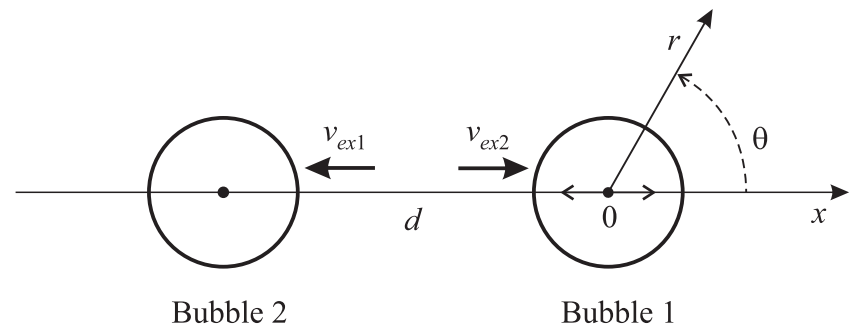

FIG. 3. Relative position of two bubbles: top view. 
well. This fact should be taken into account when calculating $a, \boldsymbol{v}_{e x}$, and $p_{\text {ex }}$.

Assuming that $j=1$ or 2 , let us denote the equilibrium radius of the $j$ th bubble by $R_{j 0}$, the dimensionless pulsation amplitude of the $j$ th bubble by $a_{j}$, and the velocity and the pressure produced by the $j$ th bubble at the position of the other bubble by $\boldsymbol{v}_{\text {exj }}$ and $p_{\text {exj }}$. In terms of these designations, the previous designations are expressed as follows: $R_{0}=R_{10}$, $a=a_{1}, \boldsymbol{v}_{e x}=\boldsymbol{v}_{e x 2}$, and $p_{e x}=p_{e x 2}$.

By using the results of Ref. [34], $p_{\text {exj }}$ can be written as

$$
p_{e x j}=\beta_{j} a_{j},
$$

where

$$
\begin{gathered}
\beta_{j}=-\frac{\rho_{f} \omega^{2}}{\pi \mu}\left(\frac{H_{0}^{(1)}\left(k_{f} d\right)}{k_{f} H_{1}^{(1)}\left(k_{f} R_{j 0}\right)}\left[\pi \mu R_{j 0}-k_{t} q_{j} I_{f r}\left(R_{j 0}, 0\right)\right]\right. \\
\left.+q_{j} I_{p}(d, 0)\right) \\
q_{j}=-2 \pi \gamma\left(P_{0}+\frac{\sigma_{f}}{R_{j 0}}\right) R_{j 0}^{2} \varepsilon_{j} \\
\varepsilon_{j}=\varepsilon_{j 1} \exp \left(i \varepsilon_{j 2} k_{s} R_{j 0}\right)
\end{gathered}
$$

Here, $\mu$ is the shear modulus of the wall material, $k_{f}=$ $\omega / c_{f}, c_{f}$ is the speed of sound in the fluid, and $k_{t}=\omega / c_{t}$, where $c_{t}$ is the speed of the transverse wave in the walls [33]. The functions $I_{f r}$ and $I_{p}$ are defined by Eqs. (2.65) and (2.67) in Ref. [34]. The quantities $\varepsilon_{j 1}$ and $\varepsilon_{j 2}$ serve as fitting parameters. They were introduced in Ref. [34] to describe the action of the bubble on the elastic walls. Finally, $k_{s}=\omega / c_{s}$, where $c_{s}$ is the speed of the surface waves propagating along the fluidsolid interfaces. It is calculated from Eq. (2.57) in Ref. [34]. Equations (95)-(98) follow from Eqs. (2.62), (2.73), (2.76), (2.87), and (3.1) in Ref. [34].

According to Eq. (2.88) in Ref. [34], the pulsation amplitude of the $j$ th bubble, with allowance made for the presence of the other bubble, can be represented as

$$
a_{j}=-\frac{P_{a}+p_{e x(3-j)}}{D_{j}},
$$

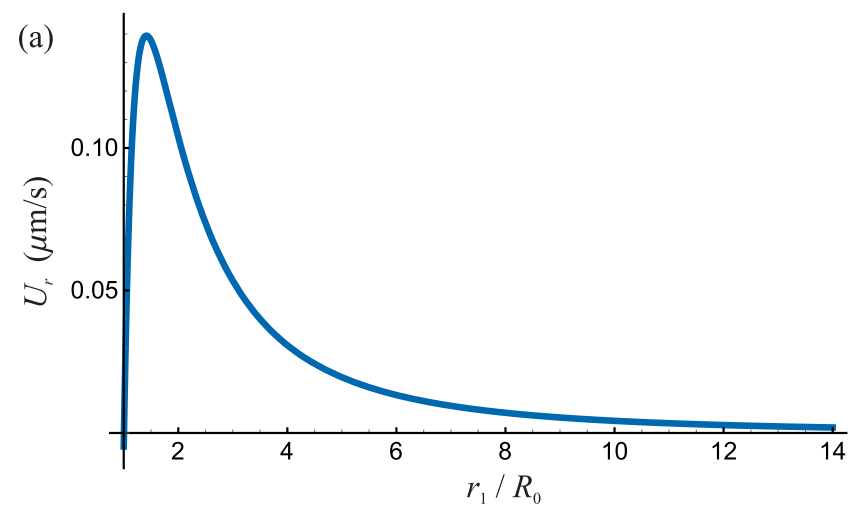

where

$$
\begin{aligned}
D_{j}= & 2 \gamma P_{0}+\frac{(2 \gamma-1) \sigma_{f}}{R_{j 0}}-\frac{\rho_{f} \omega^{2} R_{j 0}^{2} H_{0}^{(1)}\left(\alpha_{f j}\right)}{\alpha_{f j} H_{1}^{(1)}\left(\alpha_{f j}\right)} \\
& +\frac{2 \gamma \rho_{f} \alpha_{t j}^{2} \varepsilon_{j}}{\rho_{s}}\left(P_{0}+\frac{\sigma_{f}}{R_{j 0}}\right) \\
& \times\left(I_{p}\left(R_{j 0}, 0\right)-\frac{\alpha_{t j} H_{0}^{(1)}\left(\alpha_{f j}\right)}{\alpha_{f j} H_{1}^{(1)}\left(\alpha_{f j}\right)} I_{f r}\left(R_{j 0}, 0\right)\right),
\end{aligned}
$$

$\alpha_{f j}=k_{f} R_{j 0}, \alpha_{t j}=k_{t} R_{j 0}$, and $\rho_{s}$ is the wall density. Substituting Eq. (95) into Eq. (99) and solving for $a_{j}$, one obtains

$$
a_{j}=\frac{\left(\beta_{3-j}-D_{3-j}\right) P_{a}}{D_{1} D_{2}-\beta_{1} \beta_{2}} .
$$

It remains to find $\boldsymbol{v}_{e x j}$. To this end, we use Eqs. (2.60), (2.71), (2.72), (2.76), and (2.87) in Ref. [34]. As a result, we obtain the following equation for the amplitude of $\boldsymbol{v}_{\text {exj }}$ :

$$
\begin{aligned}
v_{e x j}= & \frac{i(-1)^{j+1} \omega a_{j}}{\pi \mu}\left(k_{t} q_{j} I_{f r}(d, 0)\right. \\
& \left.+\frac{H_{1}^{(1)}\left(k_{f} d\right)}{H_{1}^{(1)}\left(\alpha_{f j}\right)}\left[\pi \mu R_{j 0}-k_{t} q_{j} I_{f r}\left(R_{j 0}, 0\right)\right]\right) .
\end{aligned}
$$

To sum up, the solutions of the equations of acoustic streaming derived in our work are applicable to both bubbles. Ultimately they are expressed in terms of the pulsation amplitude of the $j$ th bubble, $a_{j}$, and the fluid velocity produced by the other bubble, $\boldsymbol{v}_{\text {ex }(3-j)}$. Therefore, to apply the solutions to the $j$ th bubble, it is necessary to substitute appropriate expressions for $a_{j}$ and $\boldsymbol{v}_{\operatorname{ex}(3-j)}$ derived in this subsection. Doing so, it should be also remembered that the cylindrical coordinates $r$ and $\theta$ appearing in the solutions should be considered as coordinates originated at the center of the $j$ th bubble. The total streaming produced by both bubbles can be evaluated assuming the additivity of this effect, namely, that the total streaming is a sum of the flows produced by the bubbles.

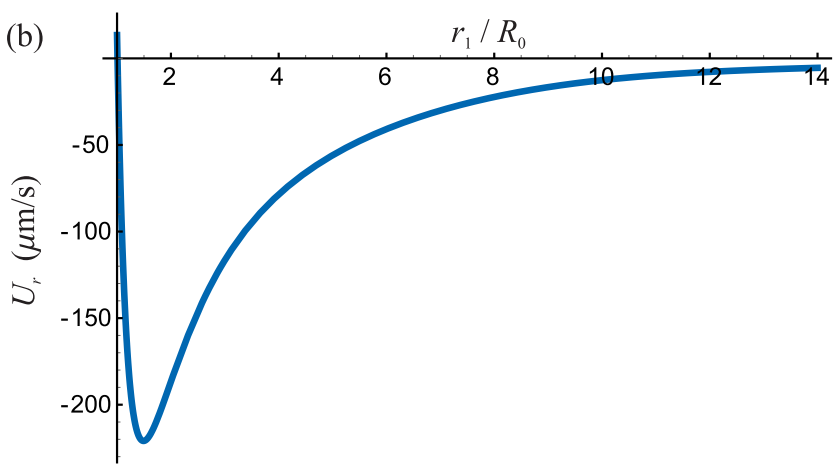

FIG. 4. The radial component of the streaming velocity at $\theta=0$ vs distance $r_{1}$ from the center of bubble 1 . The results were obtained for two equal bubbles with radii $R_{0}=30 \mu \mathrm{m}$, separated by a distance $d=10 R_{0}$ and excited at a frequency $f=50 \mathrm{kHz}$. (a) Surface waves are absent. (b) Surface waves are present. 


\section{SIMULATIONS AND EXPERIMENTAL VERIFICATION}

\section{A. Numerical examples}

We assume that the fluid in the microfluidic channel is water and the gas in bubbles is air with the following physical parameters: $\rho_{f}=998 \mathrm{~kg} / \mathrm{m}^{3}, \eta_{f}=0.001 \mathrm{Pas}, \sigma_{f}=$ $0.072 \mathrm{~N} / \mathrm{m}, c_{f}=1481 \mathrm{~m} / \mathrm{s}, P_{0}=101.3 \mathrm{kPa}$, and $\gamma=1.4$. The channel walls are assumed to be made of a polydimethylsiloxane (PDMS) elastomer the parameters of which are the density $\rho_{s}=970 \mathrm{~kg} / \mathrm{m}^{3}$, Young's modulus $E=1.6 \mathrm{MPa}$, and Poisson's ratio $\sigma=0.499$. The channel height is taken to be $h=25 \mu \mathrm{m}$ and the acoustic pressure amplitude is set equal to $P_{a}=10 \mathrm{kPa}$. To make numerical calculations easier, we consider examples with $\delta_{v} / R_{0} \ll 1$, which allows us to use Eqs. (91) and (92).

Figures 4-6 compare acoustic streaming in the cases of the absence and the presence of surface waves. The absence of surface waves implies rigid walls. The simulations were
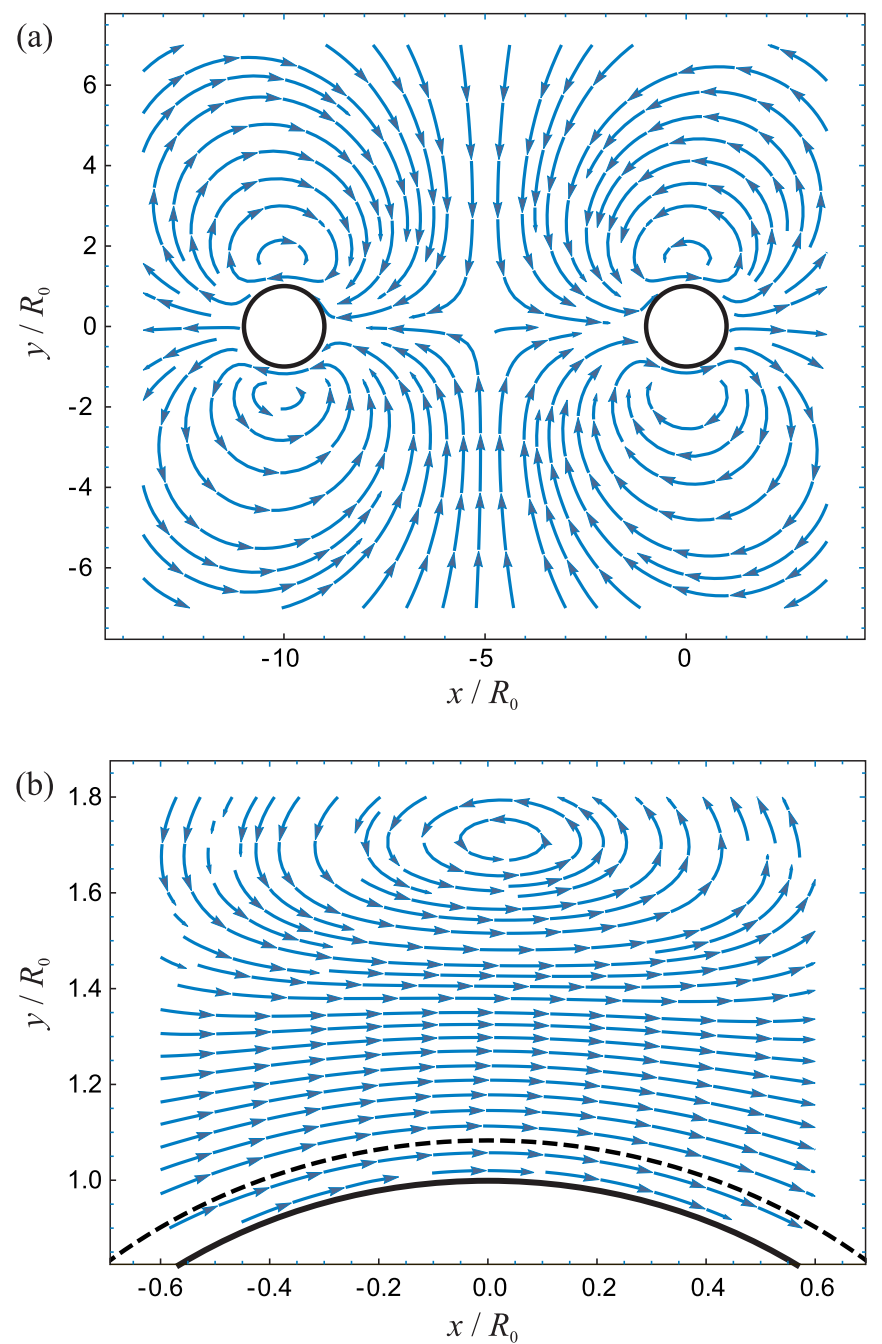

FIG. 5. Streamlines for two equal bubbles undergoing radial and translational oscillations between two walls when surface waves are absent: (a) overall view, (b) enlarged view near the surface of bubble 1 (solid line). $R_{0}=30 \mu \mathrm{m}, d=10 R_{0}, f=50 \mathrm{kHz}, \sin (\Delta \phi)=0.003$. The dashed line indicates the thickness of the viscous boundary layer $\delta_{v}=0.08 R_{0}$. The streaming is of the fountain type. made for two equal bubbles with radii $R_{j 0}=R_{0}=30 \mu \mathrm{m}$, separated by a distance $d=10 R_{0}$ and excited at a frequency $f=50 \mathrm{kHz}$. The bubble pulsation amplitudes were calculated at $\varepsilon_{j 1}=1.95$ and $\varepsilon_{j 2}=-0.756$. These values were adopted from Ref. [34]. Figure 4 shows the radial component of the total streaming velocity along the direction $\theta=0$. This quantity is calculated as $U_{r}\left(r_{1}\right)=U_{r 1}\left(r_{1}\right)+U_{r 2}\left(d+r_{1}\right)$, where $U_{r j}$ is the radial component of the velocity field produced by the $j$ th bubble and $r_{1}$ is the distance from the center of bubble 1 . In Fig. 4(a), the surface waves are absent, while in Fig. 4(b), they are present. It is seen that the surface waves change the sign of the streaming velocity and greatly increase its magnitude. Figures 5 and 6 demonstrate streamlines when the surface waves are absent and when they are present. Figures 5(a) and 6(a) depict the overall view of the streamlines and Figs. 5(b) and 6(b) show the behavior of the streamlines near the surface of bubble 1 (solid line) around the angle $\theta=\pi / 2$. The dashed line indicates the thickness of the viscous boundary
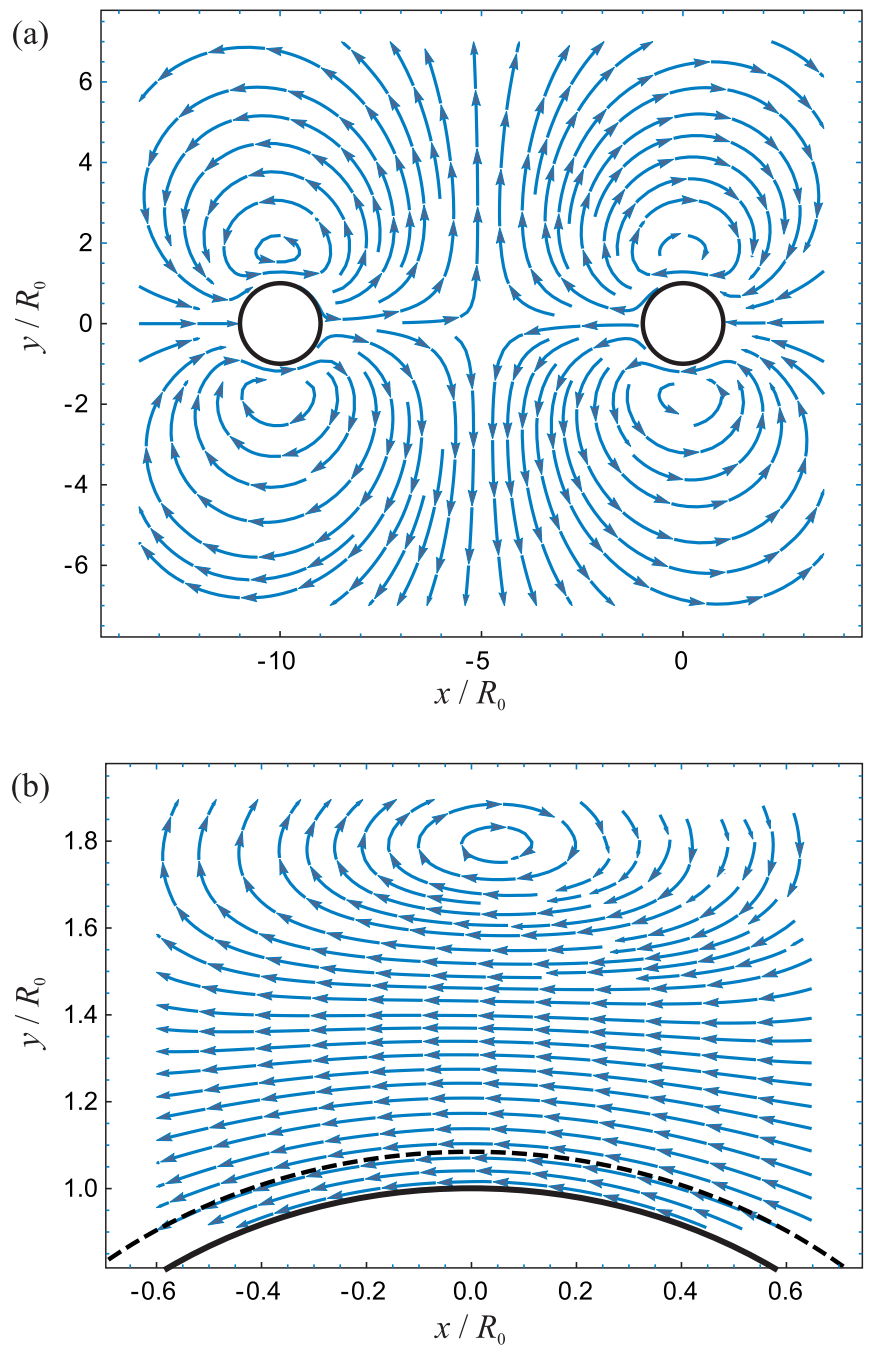

FIG. 6. Streamlines for two equal bubbles undergoing radial and translational oscillations between two walls when surface waves are present: (a) overall view, (b) enlarged view near the surface of bubble 1 (solid line). $R_{0}=30 \mu \mathrm{m}, d=10 R_{0}, f=50 \mathrm{kHz}, \sin (\Delta \phi)=-0.67$. The dashed line indicates the thickness of the viscous boundary layer $\delta_{v}=0.08 R_{0}$. The streaming is of the antifountain type. 

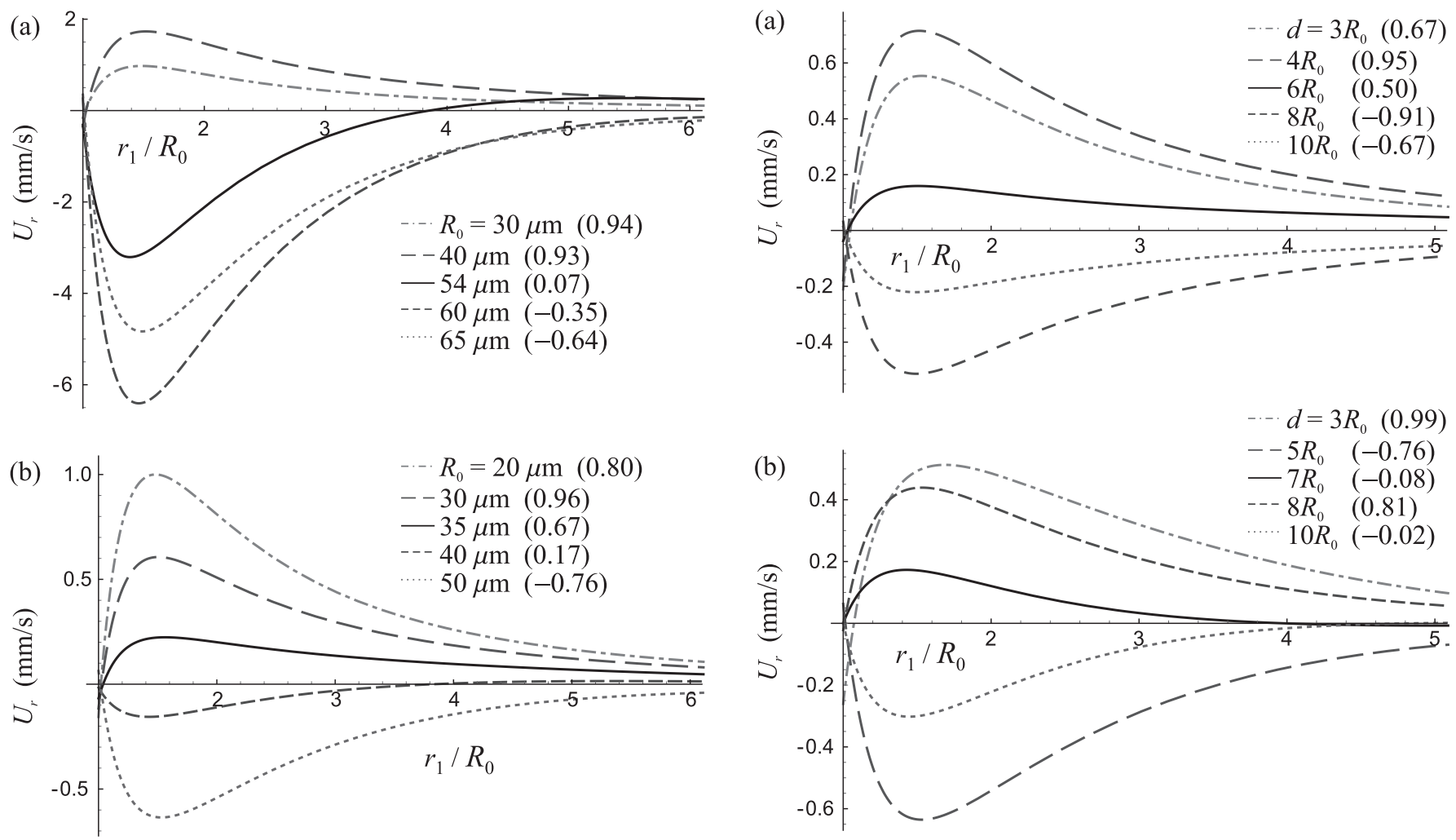

FIG. 7. Behavior of the radial component of the streaming velocity at different bubble radii. The calculations were made for two bubbles of equal radii $R_{0}$ at $\theta=0, d=5 R_{0}$ and $f=$ (a) $30 \mathrm{kHz}$ and (b) $50 \mathrm{kHz}$. $r_{1}$ is the distance from the center of bubble 1 . The numbers in brackets are the values of $\sin (\Delta \phi)$.

layer $\delta_{v}=0.08 R_{0}$. Note also that in Fig. $5, \sin (\Delta \phi)=0.003$, and in Fig. $6, \sin (\Delta \phi)=-0.67$. According to the terminology proposed by Rallabandi et al. [30], the vortex pattern in Fig. 5 is a streaming of the "fountain" type, while that in Fig. 6 is a streaming of the "antifountain" type. Thus, one can say that the surface waves reverse the vortex orientation. It is also interesting to note that for each bubble taken separately, the streamline structure resembles the flow generated by a source dipole [40].

Figures 7 and 8 demonstrate the effect of different parameters on acoustic streaming. Figure 7 shows the behavior of the radial velocity component at different values of bubble radii $R_{0}$. The velocity component is calculated along the direction $\theta=0$ at $d=5 R_{0}$ and $f=30$ and $50 \mathrm{kHz}$. The numbers in brackets are the values of $\sin (\Delta \phi)$. Note that the positive sign of $U_{r}$ corresponds to the fountain type of streaming and vice versa. Thus, one can see that, for smaller bubbles, a fountain-type streaming occurs, while for bigger bubbles, the vortex pattern changes to the antifountain type. These results conform to experimental observations reported by Mekki-Berrada et al. [32]. Comparison of Figs. 7(a) and 7(b) also reveals that the value of $R_{0}$ at which the changeover of the vortex orientation occurs decreases with increasing frequency. Figure 8 shows the behavior of the radial velocity component at different values of the separation distance $d$ between the bubbles. The calculations were made at $f=50 \mathrm{kHz}$ for $R_{0}=30 \mu \mathrm{m}$ and $50 \mu \mathrm{m}$. The numbers in brackets are the

FIG. 8. Behavior of the radial component of the streaming velocity at different interbubble distances $d$. The calculations were made for two bubbles with equal radii $R_{0}=$ (a) $30 \mu \mathrm{m}$ and (b) $50 \mu \mathrm{m}$ at $\theta=0$ and $f=50 \mathrm{kHz}$. $r_{1}$ is the distance from the center of bubble 1. The numbers in brackets are the values of $\sin (\Delta \phi)$.

values of $\sin (\Delta \phi)$. Figure 8 (a) reveals that for $R_{0}=30 \mu \mathrm{m}$, the streaming changes from the antifountain type to the fountain type as $d$ reduces from $10 R_{0}$ to $3 R_{0}$. For $R_{0}=50 \mu \mathrm{m}$, as Fig. 8(b) shows, the streaming type changes three times with decreasing $d$ : antifountain $\left(d=10 R_{0}\right)$, fountain $\left(d=7 R_{0}\right)$, antifountain $\left(d=5 R_{0}\right)$, and again fountain $\left(d=3 R_{0}\right)$.

Figures 7 and 8 suggest that in many cases, the streaming type is determined by the $\operatorname{sign}$ of $\sin (\Delta \phi): \sin (\Delta \phi)>0$ results in the fountain type, whereas $\sin (\Delta \phi)<0$ leads to the antifountain type. However, this is not always the case. For example, the curve for $R_{0}=54 \mu \mathrm{m}$ in Fig. 7(a) and the curve for $R_{0}=40 \mu \mathrm{m}$ in Fig. 7(b) show the antifountain behavior, although $\sin (\Delta \phi)>0$. On the contrary, the curve for $d=7 R_{0}$ in Fig. 8(b) shows the fountain behavior at $\sin (\Delta \phi)<0$. These examples reflect the fact that for $v_{s} \neq 0$, the dependence on $\sin (\Delta \phi)$ is not linear.

\section{B. Experimental verification}

Experimental and theoretical results are compared in Fig. 9. The experimental data are shown by circles. They were obtained using a microfluidic setup described in detail in Refs. [3] and [32]. Figure 9 shows the radial velocity component of acoustic streaming generated by a pair of bubbles. The velocity component is measured along the direction $\theta=0$ as a function of distance $r_{1}$ from the center of the right bubble (bubble 1); see the insets in Fig. 9. The data in Fig. 9(a) were obtained 

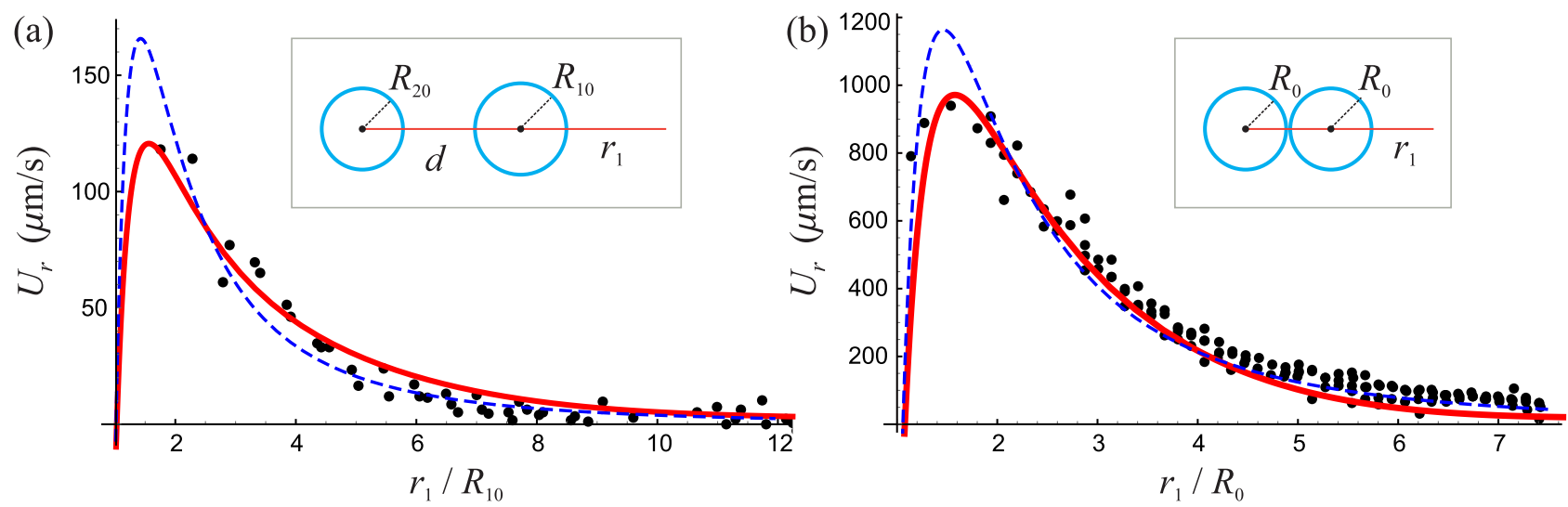

FIG. 9. Comparison of experimental (circles) and theoretical (solid and dashed lines) results. The radial component of the streaming velocity is plotted as a function of distance $r_{1}$ from the center of the right bubble (bubble 1). (a) Bubbles with radii $R_{10}=38.3 \mu \mathrm{m}$ and $R_{20}=33.6 \mu \mathrm{m}$, separated by a distance $d=175 \mu \mathrm{m}$, excited at $40 \mathrm{kHz}$. (b) Bubbles in contact, with radii $R_{0}=30 \mu \mathrm{m}$, excited at $80 \mathrm{kHz}$. The solid and dashed lines show the results given by the present model and the model of Mekki-Berrada et al. [32], respectively.

for bubbles with radii $R_{10}=38.3 \mu \mathrm{m}$ and $R_{20}=33.6 \mu \mathrm{m}$, separated by a distance of $175 \mu \mathrm{m}$ and excited at $40 \mathrm{kHz}$. The data in Fig. 9(b) were obtained for two bubbles with equal radii $R_{0}=30 \mu \mathrm{m}$, which were in contact and excited at $80 \mathrm{kHz}$. Experimental conditions do not allow one to determine the amplitude of the driving acoustic pressure, $P_{a}$. Therefore, this quantity is an adjustable parameter in simulations. It should be also mentioned that in the experiment, a surfactant was added to the fluid to prevent bubble coalescence. However, its concentration was low so the slip boundary condition on the bubble surface, applied in our theory, is justified.

The solid lines in Fig. 9 show the fitting of the experimental points by Eq. (91). The physical parameters of the fluid and the channel walls used in the simulations are the same as in the preceding subsection. The solid curve in Fig. 9(a) was calculated at $P_{a}=1.88 \mathrm{kPa}, \varepsilon_{j 1}=2.15$, and $\varepsilon_{j 2}=-0.42$. The solid curve in Fig. 9(b) was calculated at $P_{a}=34.4 \mathrm{kPa}$, $\varepsilon_{j 1}=1.95$, and $\varepsilon_{j 2}=-0.8$. The values of $\varepsilon_{j 1}$ and $\varepsilon_{j 2}$ were adopted from Ref. [34]. For comparison, the dashed lines in Fig. 9 show the results given by the model developed by Mekki-Berrada et al. [32]. The dashed curve in Fig. 9(a) was calculated at $P_{a}=10 \mathrm{kPa}$, and that in Fig. 9(b) at $P_{a}=160 \mathrm{kPa}$. Note that the model of Mekki-Berrada et al. [32] requires much higher values of the acoustic pressure. The difference between the solid and dashed curves is explained by the fact that Mekki-Berrada et al. [32] have used a simplified model for the bubble dynamics. Figure 9 shows that there is a good agreement between the experimental measurements and the theory developed in the present study.

\section{CONCLUSION}

A theoretical model has been developed for acoustic streaming generated by a cylindrical bubble squeezed in a microfluidic channel between two planar elastic walls. The model assumes that the acoustic streaming results from the mixed-mode interaction between the volume and translational modes of the bubble oscillations. The translational oscillation is supposed to originate from an external sound source such as a neighboring bubble. Unlike the previous study [34], which used a phenomenological model for bubble oscillation and ignored the existence of the bulk scattered wave, the present study is based on rigorous mathematical solutions and takes into account the existence of both surface waves propagating along the fluid-wall interfaces and the bulk scattered wave propagating in the fluid gap. The general analytical solutions to the linear equations of fluid motion and to the equations of acoustic streaming are calculated with no restriction on the ratio between the viscous penetration depth and the bubble size. Approximate solutions for the limit of low viscosity are provided as well. Numerical simulations of acoustic streaming generated by bubble pairs are performed. They show that the vortex orientation of streamline patterns is governed by the driving frequency, bubble size, and the interbubble distance. Experimental verification of the developed theory has been performed using data for streaming generated by bubble pairs. It demonstrated good agreement between the theoretical predictions and the experimental measurements. The obtained results are of immediate interest for microfluidic investigations.

\section{ACKNOWLEDGMENTS}

This research has received funding from the European Research Council under the European Union's Seventh Framework Programme (FP7/2007-2013)/ERC Grant Agreement No. 614655 "Bubbleboost".
[1] P. Marmottant, J. P. Raven, H. Gardeniers, J. G. Bomer, and S. Hilgenfeldt, J. Fluid Mech. 568, 109 (2006).

[2] J. Friend and L. Yeo, Rev. Mod. Phys. 83, 647 (2011).
[3] D. Rabaud, P. Thibault, M. Mathieu, and P. Marmottant, Phys. Rev. Lett. 106, 134501 (2011).

[4] S. J. Lighthill, J. Sound Vibr. 61, 391 (1978). 
[5] M. S. Longuet-Higgins, Philos. Trans. R. Soc. London, Ser. A 245, 535 (1953).

[6] N. Riley, Annu. Rev. Fluid Mech. 33, 43 (2001).

[7] B. R. Lutz, J. Chen, and D. T. Schwartz, Anal. Chem. 78, 5429 (2006).

[8] C. Wang, S. V. Jalikop, and S. Hilgenfeldt, Appl. Phys. Lett. 99, 034101 (2011).

[9] P. Rogers and A. Neild, Lab Chip 11, 3710 (2011).

[10] T. Franke, S. Braunmüller, L. Schmid, A. Wixforth, and D. Weitz, Lab Chip 10, 789 (2010).

[11] C. Wang, S. V. Jalikop, and S. Hilgenfeldt, Biomicrofluidics 6 , 012801 (2012).

[12] P. Marmottant and S. Hilgenfeldt, Nature (London) 423, 153 (2003).

[13] R. H. Liu, J. Yang, M. Z. Pindera, M. Athavale, and P. Grodzinski, Lab Chip 2, 151 (2002).

[14] D. Ahmed, X. Mao, J. Shi, B. K. Juluri, and T. J. Huang, Lab Chip 9, 2738 (2009).

[15] S. S. Wang, Z. J. Jiao, X. Y. Huang, C. Yang, and N. T. Nguyen, Microfluid. Nanofluid. 6, 847 (2009).

[16] C. Wang, B. Rallabandi, and S. Hilgenfeldt, Phys. Fluids 25, 022002 (2013).

[17] J. Kolb and W. Nyborg, J. Acoust. Soc. Am. 28, 1237 (1956).

[18] W. L. Nyborg, J. Acoust. Soc. Am. 30, 329 (1958).

[19] S. A. Elder, J. Acoust. Soc. Am. 31, 54 (1959).

[20] N. Riley, Q. J. Mech. Appl. Math. 19, 461 (1966).

[21] N. Riley, IMA J. Appl. Math. 3, 419 (1967).

[22] B. J. Davidson and N. Riley, J. Sound Vib. 15, 217 (1971).

[23] J. Wu and G. Du, J. Acoust. Soc. Am. 101, 1899 (1997).

[24] M. S. Longuet-Higgins, Proc. R. Soc. London, Ser. A 454, 725 (1998).
[25] X. Liu and J. Wu, J. Acoust. Soc. Am. 125, 1319 (2009).

[26] A. A. Doinikov and A. Bouakaz, J. Acoust. Soc. Am. 127, 703 (2010).

[27] A. A. Doinikov and A. Bouakaz, J. Acoust. Soc. Am. 127, 1218 (2010).

[28] A. A. Doinikov and A. Bouakaz, J. Fluid Mech. 742, 425 (2014).

[29] W. P. Raney, J. C. Corelli, and P. J. Westervelt, J. Acoust. Soc. Am. 26, 1006 (1954)

[30] B. Rallabandi, C. Wang, and S. Hilgenfeldt, J. Fluid Mech. 739, 57 (2014).

[31] B. Rallabandi, A. Marin, M. Rossi, C. J. Kähler, and S. Hilgenfeldt, J. Fluid Mech. 777, 408 (2015).

[32] F. Mekki-Berrada, T. Combriat, P. Thibault, and P. Marmottant, J. Fluid Mech. 797, 851 (2016).

[33] I. A. Viktorov, Rayleigh and Lamb Waves: Physical Theory and Applications (Plenum, New York, 1967).

[34] A. A. Doinikov, F. Mekki-Berrada, P. Thibault, and P. Marmottant, Proc. R. Soc. A 472, 20160031 (2016).

[35] F. Mekki-Berrada, P. Thibault, and P. Marmottant, Phys. Fluids 28, 032004 (2016).

[36] Yu. A. Ilinskii, E. A. Zabolotskaya, T. A. Hay, and M. F. Hamilton, J. Acoust. Soc. Am. 132, 1346 (2012).

[37] M. Abramowitz and I. N. Stegun, Handbook of Mathematical Functions (Dover, New York, 1965).

[38] L. D. Landau and E. M. Lifshitz, Fluid Mechanics (Pergamon, Oxford, 1987).

[39] W. E. Boyce and R. C. DiPrima, Elementary Differential Equations and Boundary Value Problems (Wiley, New York, 2001).

[40] S. E. Spagnolie and E. Lauga, J. Fluid Mech. 700, 105 (2012). 